\title{
WATER AREA OBSERVATION EXPERIMENT USING OPTICAL SENSOR FOR EXTRACTING INUNDATION BOUNDARY AT NIGHT
}

\author{
T. Nakano ${ }^{1, *}$, R. Endo ${ }^{1}$, H. Ohno ${ }^{1}$, J. Iwahashi ${ }^{1}$ \\ ${ }^{1}$ Geospatial Information Authority of Japan, 1 Kitasato, Tsukuba, Ibaraki, Japan - \\ nakano-t96fj@mlit.go.jp
}

Commission III, WG III/IVa

KEYWORDS: night time, inundation area, water area, ultrasensitive camera, thermal infrared camera, helicopter

\begin{abstract}
:
During the occurrence of floods, the government generally tries to determine the extent of the continuously changing inundation (water) area as a disaster response for planning evacuations and designing drainage systems. One of the most common methods to identify this area during the daytime involves the interpretation of aerial optical images and videos that are obtained using an airplane, helicopter, or satellite. However, these aerial approach methods have not been established convincingly for their use during the night time. Therefore, to identify the water area at night, two hand-held optical sensors were used in this study, namely: an ultrasensitive camera (Sony SL camera $\alpha 7 \mathrm{~S}$ ), and a thermal infrared camera (Optris thermography PI640). The night-time aerial performance was evaluated using a helicopter, along with a supplementary night-time examination on the ground. Herein, we targeted river water areas during normal conditions. As a result, it was determined that the water area could be identified clearly with an ultrasensitive camera that has an ISO sensitivity between 51200 and 102400 . The thermal infrared camera was able to identify the water area to have a higher temperature than that of the surrounding area. However, in some cases, the water area boundaries were not clear. Thus, as the thermal infrared camera was susceptible to varying conditions, supplemental use of an ultrasensitive camera was determined to be effective for the identification of inundation (water) areas at night. The significance of this study is established by the fact that the studied sensors can be easily transported in a helicopter or mounted on a UAV for night-time applications.
\end{abstract}

\section{INTRODUCTION}

Large-scale flooding has recently become frequent worldwide, and Japan is no exception. When a flood occurs, the government tries to elucidate the extent of the resultant inundation area for effective disaster management. It is important to identify this continuously changing area for planning evacuations and drainage systems. In particular, it is important to know the location of the inundation front. A relatively common method to identify the inundation area during the daytime is the interpretation of the aerial optical images and videos, which are obtained using an airplane, helicopter, or satellite. Recently, automatic extraction by machine learning has also been attempted using these images. However, there are no credible methods to identify the inundation area using the aerial approach at night. One such method uses synthetic aperture radar (SAR); however, the observational timings of all the major SAR satellites are approximately twice a day, with an uncertainty in the possibility of obtaining the images at night. It is also difficult to operate the SAR on-board the Cessna aircraft at night because of the limited availability of the aircraft and the requirement of a clear vision during the flight.

Therefore, we selected an ultrasensitive camera and a thermal infrared camera as hand-held optical sensors that can be placed in a helicopter, which is relatively easy to fly during the night time; this was aimed at testing their aerial performance for detecting inundation areas at night.

\section{RESEARCH METHODOLOGY}

\subsection{Survey and selection of sensors}

Based on previous research, the following sensors have been listed as effective for the observation of water areas at night using helicopters: ultra-/high-sensitive camera, near-/farinfrared camera, thermal infrared camera (thermography), and night vision camera.

Although there are almost no examples of observational studies that were carried out at night time using an ultra-/high-sensitive camera, the purpose of this camera is to facilitate night-time photography. Furthermore, the product manufacturer confirmed that it could shoot in total darkness. In addition, closed-circuit television (CCTV) cameras for monitoring have been installed at major rivers in Japan, which comprise high-sensitivity specifications that enable night-time shooting. Therefore, for the purposes of our study, these cameras were practical.

A near-infrared camera is good at detecting water; however, it needs to actively irradiate infrared rays at night, and is not suitable for carrying out observations from altitudes over several hundred meters.

Previous experimentations for performing night-time water observations using a far-infrared camera to observe river flow have been carried out by Fujita et al. (2013) and Sasaki et al. (2018). These studies show that the method of using a farinfrared camera has reached a level of practical relevance for night-time monitoring of river flow in Japan. However, it was difficult to select suitable equipment for observation from an altitude of several hundred meters since there are only a few types of hand-held far-infrared cameras on the market and their resolutions are not high enough.

\footnotetext{
* Corresponding author.
} 
Thermal infrared cameras have been used for water area observations by Handcock et al. (2012) during the daytime and by Sasano et al. (2010) and Puleo et al. (2012) at night. The thermal infrared camera is a good candidate for our research because there are many commercially available hand-held versions and thermal infrared cameras have already been installed in some of Japan's disaster prevention helicopters; however, there is no record of their operation during night time. The night vision camera is similar to the infrared camera as there are many commercially available products; however, many of them are not suitable for this test because of their low resolution.

Consequently, we selected an ultrasensitive camera (Sony single-lens camera $\alpha 7 \mathrm{~S}$ ) and a thermal infrared camera (Optris thermography PI640) for the purpose of this study based on feasibility, ease of availability, and portability. The ultrasensitive camera has a maximum ISO sensitivity of 409600, an effective pixel of approximately 24.3 million pixels, and a high-power zoom lens. The thermal infrared camera can observe temperatures from -20 to $100{ }^{\circ} \mathrm{C}$, and has a wide-angle lens (viewing angle of $90^{\circ}$ ) as well as a telephoto lens (viewing angle of $15^{\circ}$ ). The theoretical instantaneous field of view (IFOV) of each lens were approximately $22.1 \mathrm{~cm}$ and $4.1 \mathrm{~cm}$ at a $100 \mathrm{~m}$ distance, respectively.

\subsection{Night-time aerial performance test}

The observation site for our night-time aerial performance test was selected by considering the operating conditions of the helicopter used as a platform, the brightness around the water area, and the ease of approaching the water area on the ground. The selected location was the Nagaragawa River flowing through the Nobi Plain, in central Japan. Since it was operationally difficult to perform these tests during actual flood events, we observed river water areas during normal conditions. Flooding in Japan usually occurs between the summer and autumn seasons during heavy rain events. Therefore, it is desirable to test the thermal infrared camera at these times. However, it is difficult to perform night-time observations from the spring to autumn due to the relationship between the available time of airports used by helicopters and sunset. Thus, the aerial performance test had to be carried out in the winter. The aerial test was conducted from 18:00 to 19:30 on January 23, 2019 in cloudy weather on a night with no moon. The flight altitude was $500-800 \mathrm{~m}$. In this test, ground markers for each camera were installed (Figure 1) to ensure that the position on the ground could be identified on the captured images. Air, ground surface, and water temperatures were measured near the ground markers, in synchronization with the aerial observation to verify the thermal infrared camera. We observed the river water area of the observation site from the sky through the open window of the helicopter (AS350 type) (Figure 2). The ultrasensitive camera photographed obliquely with varying ISO sensitivity to find the optimal ISO sensitivity. The other settings were set automatically. The thermal infrared camera took oblique snapshots at 1-second intervals using a wide-angle lens and a telephoto lens. Moreover, to obtain the true positional data of the river water area, we captured aerial photographs by aerial survey during the daytime on the same day and used them to create a high-precision ortho-image. After acquiring the night-time oblique images, we determined whether the water area (water area boundary) could be identified in the captured images. Then, the optimal imaging conditions were extracted, and the obtained knowledge was summarized.

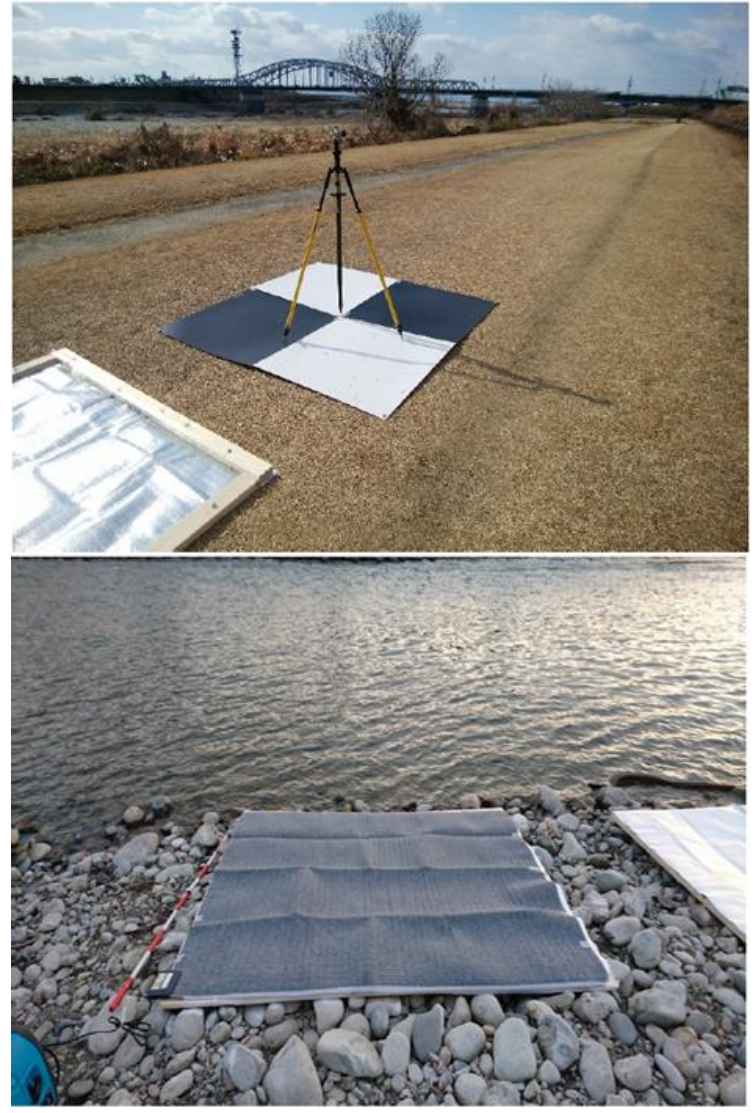

Figure 1. Left: Ground marker for ultrasensitive camera (air photo signal). Right: Ground marker for thermal infrared camera (heated floor mat of approximately $3 \mathrm{~m}$ square)

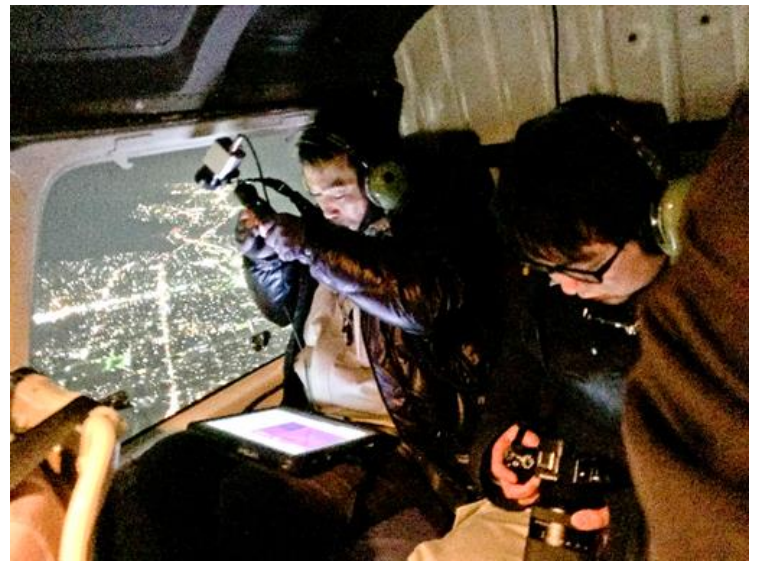

Figure 2. Observation conditions in the helicopter. The person on the left is observing with a thermal infrared camera. The person on the right is checking the images taken by the ultrasensitive camera. The helicopter window is open. 


\section{FINDINGS}

The following findings are based on the winter performance test of the ultrasensitive camera and thermal infrared camera.

\subsection{Ultrasensitive camera test}

Figure 3 shows a few of the oblique images captured with the ultrasensitive camera. At the beginning of our test, we tried the maximum ISO sensitivity of 409600; however, the grain was coarse, the resolution was poor, and it did not appear as bright as expected. Where there was a light source around the water area, it was easier to identify the water area by narrowing the shooting range rather than shooting a wide range. Furthermore, the autofocus (AF) setting did not focus well. When the focus was changed to manual focus (MF), it was confirmed that the water area could be identified even with an ISO sensitivity of 102400. We also searched for the lower limit of the ISO sensitivity, and found that even at the ISO sensitivity of 51200, the water area could be identified and the resolution increased; however, the image was blurred approximately once every few shots.

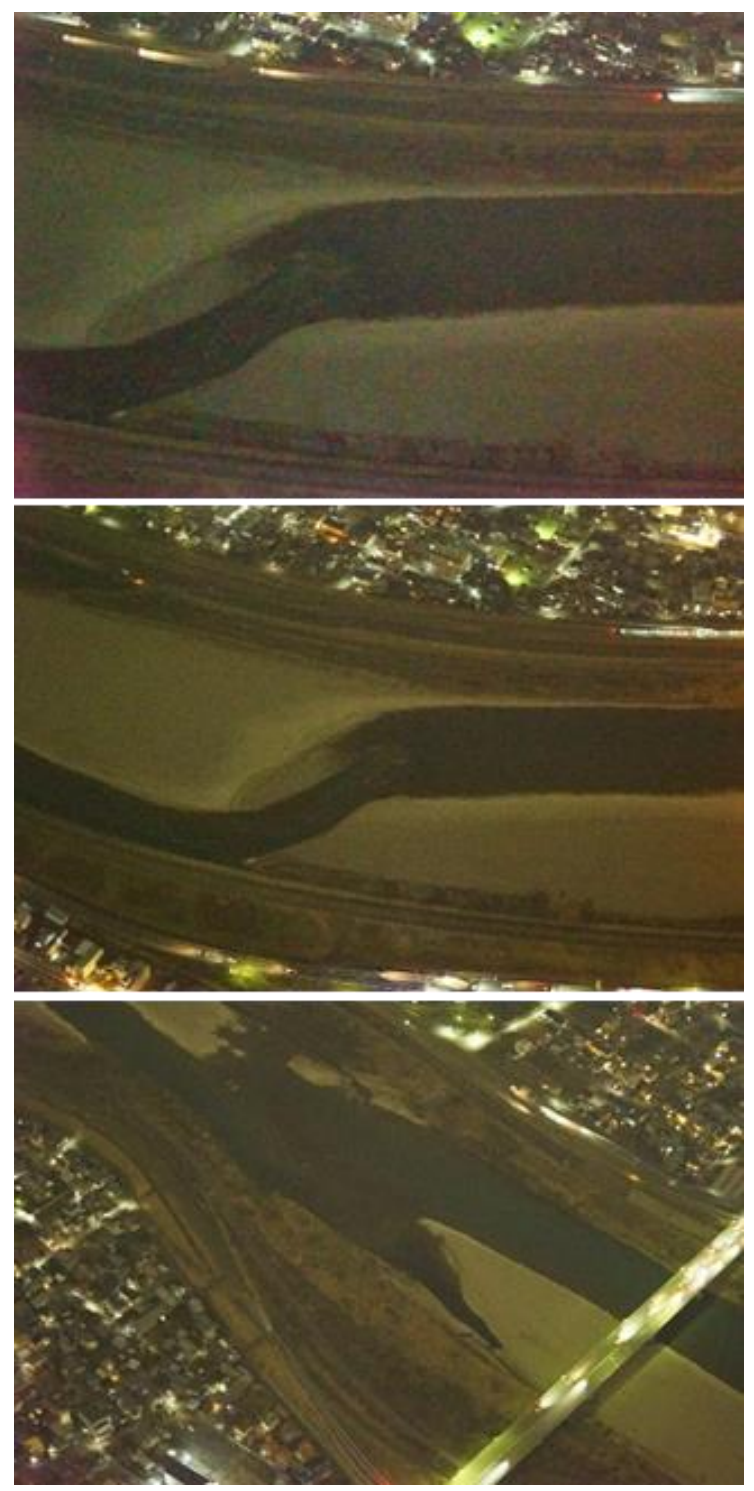

Figure 3. Ultrasensitive camera images. Top: ISO 409600, middle: ISO 102400, bottom: ISO 51200

\subsection{Thermal infrared camera test}

Figure 4 shows two of the oblique images captured with the wide-angle lens of the thermal infrared camera. The wide-angle lens was able to capture a wide area; however, when the shooting depression angle was small, the sky was reflected on the water surface and the temperature could not be measured correctly (upper image of Figure 4) because the temperature became uncharacteristically low. When the shooting depression angle was relatively large, the water area generally showed a constant range of temperatures and could be identified as a higher temperature area than the surrounding areas (lower image of Figure 4). However, the ground sampling distance was large, and the water edge was blurred.

Figure 5 shows a couple images captured with the telephoto lens of the thermal infrared camera. This figure also shows the daytime ortho images of the same location for reference. Although the telephoto lens has a small ground sampling distance, the shooting range was narrow at an altitude of 500 to $800 \mathrm{~m}$. We found that the water area could be adequately identified as a higher temperature area than the surrounding areas and the ground marker (approximately $3 \mathrm{~m}$ square) was identified clearly (lower image of Figure 5) as well. The thermal infrared camera was able to measure the temperature with an accuracy of approximately $\pm 1{ }^{\circ} \mathrm{C}$ compared with the water and ground surface temperatures measured at ground level (bottom table of Figure 5). However, in some places, the water area boundaries were unclear (lower image of Figure 5). In addition, similar temperature regions were distributed around the water area; therefore, automatic mechanical extraction based on temperature thresholds was considered difficult.

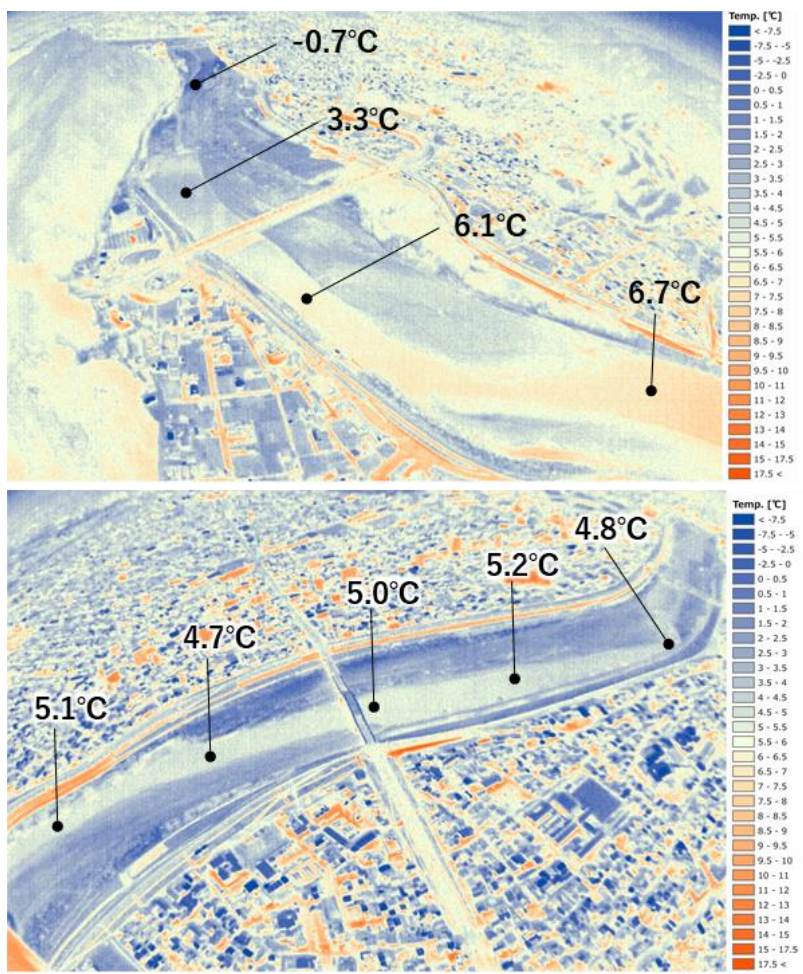

Figure 4. Thermal infrared camera images captured with the wide-angle lens. Upper: case of small shooting depression angle, lower: case of large shooting depression angle. 


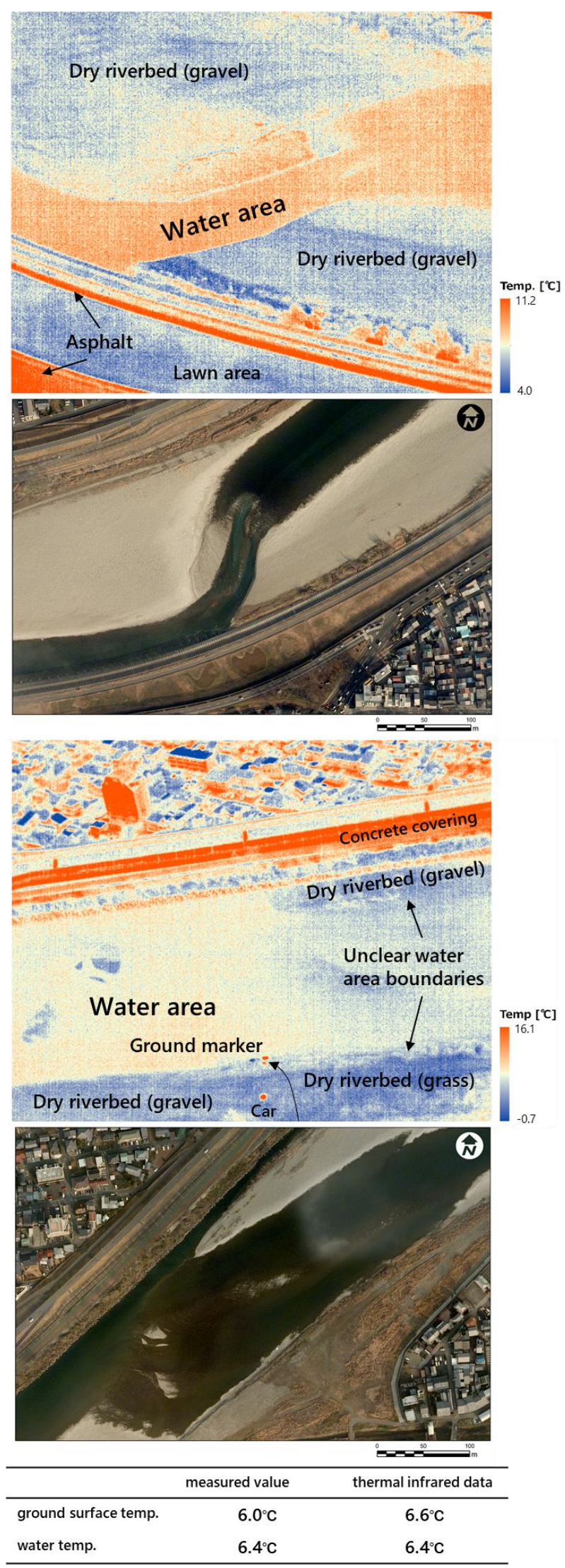

Figure 5. Thermal infrared camera images captured with the telephoto lens. The images below each temperature images are an ortho image containing the same location as the temperature image.

\subsection{Findings obtained from performance tests}

The following findings were obtained for the ultrasensitive camera:

- The optimum ISO sensitivity is between 51200 and 102400 .

- MF is easier to focus than AF.

- If there is a light source around the water area, it is easier to identify the water area by narrowing the shooting range.

The following findings were obtained for the thermal infrared camera:

- When the shooting depression angle is large (close to vertical), the temperature of the water area is within a certain range that the water area can be qualitatively identified (in the winter, it can be identified as a higher temperature area than the surrounding area). However, there are some unclear water area boundaries, and there are many similar temperature regions around it; therefore, it is currently difficult to use automatic mechanical extraction with temperature thresholds.

- A wide-angle lens is suitable for wide-area photography; however, due to the shallow depression angle, the correct temperature cannot be measured when the sky is reflected on the water surface. In addition, the ground sampling distance was large and the water boundary blurred.

- The telephoto lens has a small ground sampling distance; however, the shooting range becomes excessively narrow at an altitude of 500 to $800 \mathrm{~m}$. Therefore, a lens of intermediate viewing angle is more suitable.

\section{CONCLUSIONS}

We selected an ultrasensitive camera and a thermal infrared camera to identify water areas at night. Further, we conducted a performance test to identify the river water area from the sky during night time. As a result, the water area could be determined clearly with an ultrasensitive camera that exhibited an ISO sensitivity between 51200 and 102400 . The highest ISO sensitivity value of a single-lens reflex camera belonging to the mid-tier market segment or higher was approximately 102400; therefore, a camera with the same degree of sensitive is available easily. Although the thermal infrared camera could identify the water area, it was susceptible to varying conditions. Therefore, the supplemental use of an ultrasensitive camera was considered to be effective for the water area identification at night. These sensors offer the advantage of being carried in a helicopter or mounted on a UAV, rendering them highly practical. However, this performance evaluation is only qualitative, and it is necessary to evaluate the discrimination degree quantitatively. In addition, it is also necessary to accumulate examples of floods during summer and rainy weather, actual inundation (muddy water), and inundation cases in urban areas and paddy fields. Since the sensors used in this study do not acquire positional information and are based on oblique imaging, mechanical automatic extraction and mapping of the water area are still difficult. In the future, we plan to quantitatively evaluate the discrimination degree of the results of this test and to acquire cases at night during summer and rainy weather.

\section{ACKNOWLEDGEMENTS}

In this performance test at Nagara River, Gifu Prefecture, Gifu City, and the Nagaragawa River First Branch Office, Kisogawa River Upper Reaches River Office, Ministry of Land, 
Infrastructure, Transport and Tourism gave us permission to use river zone.

\section{REFERENCES}

Fujita, I., Kosaka, Y., Yorozuya, A. and Motonaga, Y., 2013. Surface Flow Measurement of Snow Melt Flood by Using A Far Infrared Camera. Journal of Japan Society of Civil Engineers, Ser. B1 (Hydraulic Engineering), 69(4), I_703I_708. (in Japanese with English abstract)

Handcock, N.R., Torgersen, E.C., Cherkauer, A.K., Gillespie, R.A., Tockner, K., Faux, N.R. and Tan, J., 2012. Thermal Infrared Remote Sensing of Water Temperature in Riverine Landscapes. Chapter 5 of Fluvial Remote Sensing for Science and Management, First Edition. Edited by Patrice E. Carbonneau and Hervé Piégay, 85-113.

Puleo, A.J., McKenna, E.T., Holland, T.K. and Calantoni, J., 2012. Quantifying riverine surface currents from time sequences of thermal infrared imagery. WATER RESOURCES RESEARCH, 48, W01527. doi:10.1029/2011WR010770.

Sasaki, Y., Sato, T. and Hiragaki, T., 2018. Study on applicability of image processing type flow observation Advanced flow observation for large scale water outflow (Part 3) -. Proceedings of the 61st Hokkaido Development Technology Research Meeting, 1-6. (in Japanese)

Sasano, M., Kayano, J., Futaki, Y. and Maeda, K, 2010. Development of the all-Day, all-Weather Hybrid Marine Surveillance System. The Journal of Japan Institute of Navigation, 123, 61-68. (in Japanese with English abstract) 